\title{
Design and Dynamic Model of a Frog-inspired Swimming Robot Powered by Pneumatic Muscles
}

\author{
Ji-Zhuang Fan ${ }^{1} \cdot$ Wei Zhang $^{1} \cdot$ Peng-Cheng Kong ${ }^{1} \cdot$ He-Gao Cai ${ }^{1} \cdot$ \\ Gang-Feng $\operatorname{Liu}^{1}$ (D)
}

Received: 17 January 2017/Revised: 10 April 2017/ Accepted: 24 July 2017 / Published online: 4 August 2017

(C) The Author(s) 2017. This article is an open access publication

\begin{abstract}
Pneumatic muscles with similar characteristics to biological muscles have been widely used in robots, and thus are promising drivers for frog inspired robots. However, the application and nonlinearity of the pneumatic system limit the advance. On the basis of the swimming mechanism of the frog, a frog-inspired robot based on pneumatic muscles is developed. To realize the independent tasks by the robot, a pneumatic system with internal chambers, micro air pump, and valves is implemented. The micro pump is used to maintain the pressure difference between the source and exhaust chambers. The pneumatic muscles are controlled by high-speed switch valves which can reduce the robot cost, volume, and mass. A dynamic model of the pneumatic system is established for the simulation to estimate the system, including the chamber, muscle, and pneumatic circuit models. The robot design is verified by the robot swimming experiments and the dynamic model is verified through the experiments and simulations of the pneumatic system. The simulation results are compared to analyze the functions of the source pressure, internal volume of the muscle, and circuit flow rate which is proved the main factor that limits the response of muscle pressure. The proposed research provides the application of the pneumatic muscles in the frog inspired robot and the pneumatic model to study muscle controller.
\end{abstract}

Supported by National Natural Science Foundation of China (Grant No. 51675124).

Gang-Feng Liu

liugangfeng@hit.edu.cn

1 State Key Laboratory of Robotics and System, Harbin Institute of Technology, Harbin 150080, China
Keywords Frog-inspired robot $\cdot$ Pneumatic muscle $\cdot$ Highspeed switch valve $\cdot$ Pneumatic model

\section{Introduction}

The increasing demand for underwater exploration and development, water detection, and other works in the scientific and military fields [1] has led to the rapid development of bionic underwater robots [2]. These robots mimic animal locomotion mechanisms, such as biological motion and musculoskeletal characteristics, which have been the focus of several studies [3, 4]. With regard to underwater swimming methods, most research has focused on the waving and oscillating propulsion of fishes, jet propulsion in squids, multi-legged crawling propulsion, hydrofoil flapping propulsion in turtles, and swimming motions of frogs [5-10].

Frogs brilliantly possess land jumping and underwater swimming abilities [11], and thus greatly inspired relevant robot designs in bionic research. The amphibious robot can serve in an extended wide terrain to complete tasks. Therefore, frog inspired robot is a promising topic to mimic its swimming and jumping movements which are accomplished by the same hind leg. However, current research focuses on the jumping prototype and mechanism of swimming, while the development of frog-inspired swimming prototype is less studied.

Swimming mechanisms have been widely investigated through the analysis of motion characteristics [12], propulsive force [13], and flow field structures [14]. Using experimental observations, Pandey et al. established a CAD model of a bionic frog swimming robot to mimic biological frog motions [15]. 
Frog swimming is intermittent and shows explosive movements. Thus, the driver must have high output power and fast response. Pneumatic artificial muscles have highpower mass ratio and flexible characteristics, and are similar with biological muscles in terms of driving the skeletal system [16]. In the present study, the frog-inspired robot is driven by pneumatic muscles that simulate the performance of biological frog muscles. The similarities between the pneumatic and biological muscles must be determined and analyzed to understand the relationship between the amphibious movements and musculoskeletal system of frogs.

Traditional drivers, such as motors, run through complex mechanisms [17]. Meanwhile pneumatic muscles have been widely used because of its simple assembly [18] and are mainly controlled by tracking control methods [19-21]. Several studies have performed on pneumatic muscles, mainly focusing on the modeling and control of pneumatic valves [22-24]. The precise pressure proportional valve is typically used to achieve output accuracy, but the cost is expensive and the flow rate of the pressure proportional valve is relatively small. Further, such setup is not suited for situations demanding large flow inputs. In this paper, pneumatic muscles are controlled by high-speed switch valves, which have high flow rate and fast response, thereby simplifying the design of the frog-inspired robot. In addition, high-speed switch valves are light weight, occupy small volume, and incur low cost, and is thus preferable in the robot system.

To mimic frog swimming performance, a frog inspired swimming robot is developed with pneumatic muscles and high-speed switch valves to study pneumatic characteristics during leg extension.

\section{Design of Frog-inspired Robot}

\subsection{Modeling of the Frog-inspired Robot}

Figure 1 shows the model of the frog-inspired robot, which is based on the morphology of real frogs. The palms were modeled as flexible planes with embedded spokes. The forelegs were relatively small compared with the thick and strong hind legs, which contribute slightly to the swimming propulsion. Therefore, forelegs were disregarded in the design. Considering that all the limbs moved in planar space, frog joints were modeled as planar revolute joints. The palms are main propellers for swimming, and thus, the multi DOF hind legs could be simplified as a three-DOF link mechanism for each hind leg. The entire frog model consists of the body, thigh, crus, and palm connected by the hip, knee, and ankle joints, respectively.

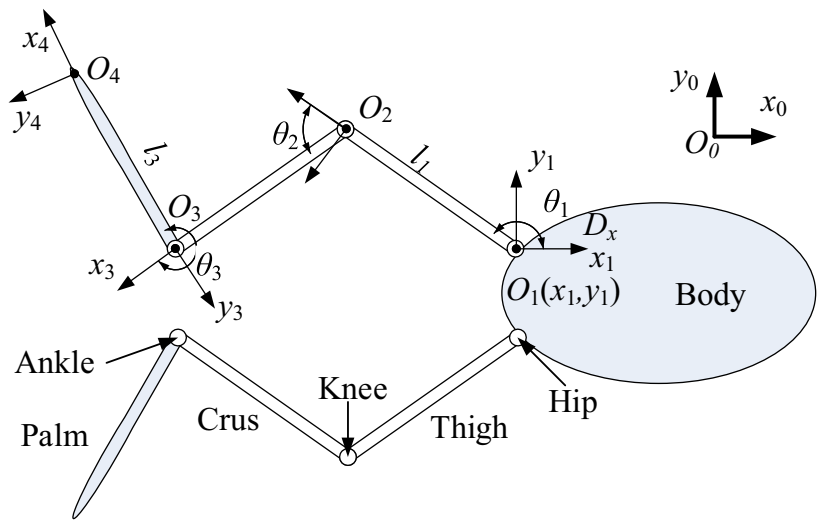

Figure 1 Model of the frog-inspired robot

\subsection{Structure of the Frog-inspired Robot}

\subsubsection{Robot System}

The major pneumatic system is designed inside the robot. Figure 2 shows that the pneumatic units are integrated in the robot body, but several pneumatic muscles are in the hind legs. A small upper shell and large bottom shell are separated by a metal baffle plate on which two cylinder
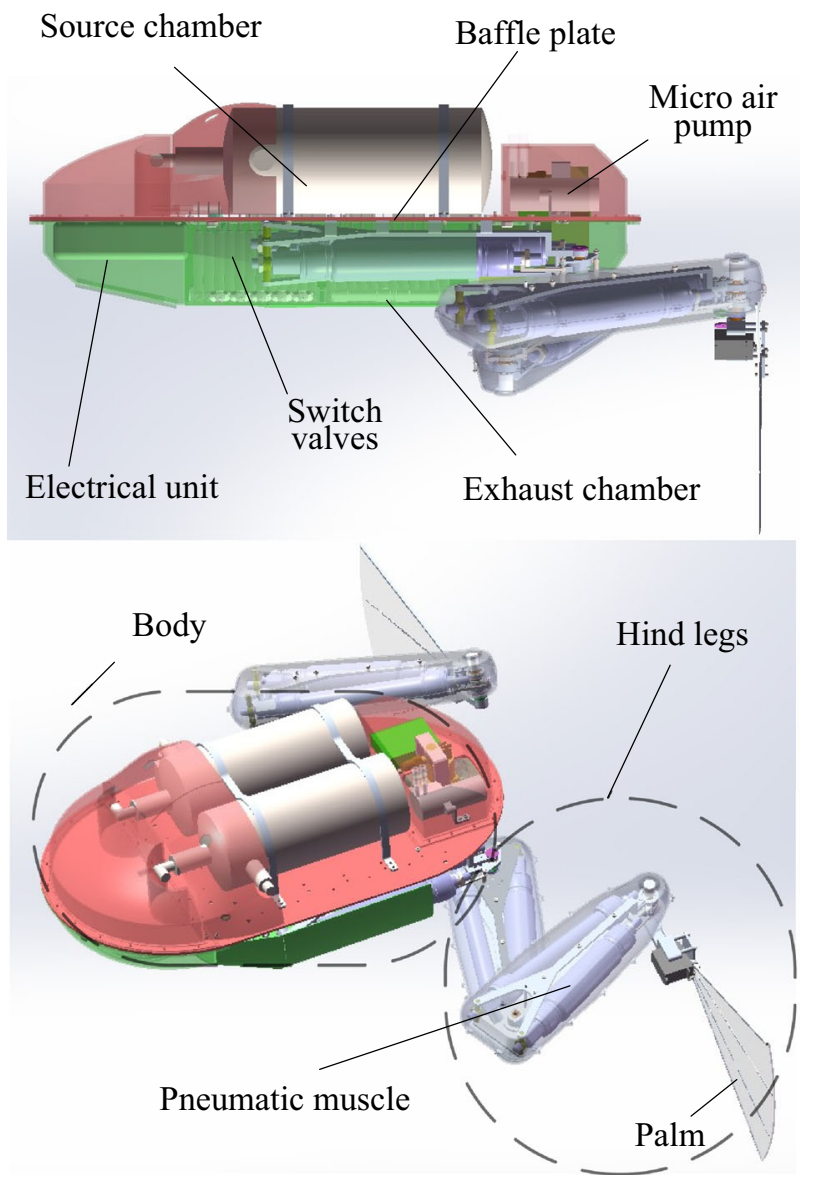

Figure 2 Structure of the frog-inspired robot 
tanks of $2 \mathrm{~L}$ each are placed as source chambers. The switch valves are installed inside the exhaust chamber and connect muscles and the source and exhaust chambers by air pipes. The body and legs are sealed by the shells, while the joints are dynamically sealed according to the joint design in the following section.

A micro air pump is placed in the rear trunk. Meanwhile, given the size of the air pump, we make it pass through the baffle plate connected to the cooling fin of the pump. The source chamber is connected to the outlet of the pump and filled with high-pressure compressed air. The high-speed switch valves are mounted in the middle of the baffle plate on the bottom face. The electrical unit is located in the front head of the exhaust chamber, which is also connected to the inlet of the pump. Therefore, the switch valve controls the flow from the source chamber to the muscle for pressurizing and from the muscle to the exhaust chamber for depressurizing.

\subsubsection{Design of the Hind Legs}

The robot is designed according to the model in this paper. Each leg (Figure 3) is framed by two upper and lower Y-shaped skeletons connected to one end of the pneumatic muscle. The other end is connected to the shaft via a crank to enable the muscle to rotate on the joint. To realize the seal for the leg and dynamic seal for the joint, the thigh and crus are covered by the shells and separately laminated on the shaft. The crus is then fixed with the shaft to enable the crus to rotate relative to the thigh through the thigh muscle drives.

Each joint has two muscles mounted in an antagonistic way to control the angle position and joint stiffness. For the knee and ankle joints, we selected the pneumatic muscle of DMSP-20-150N-RM-CM, whose length is $150 \mathrm{~mm}$ and has a maximum contraction rate of $25 \%$ and maximum contractile force of $1500 \mathrm{~N}$. We selected those with lengths of $180 \mathrm{~mm}$ for the hip joints.

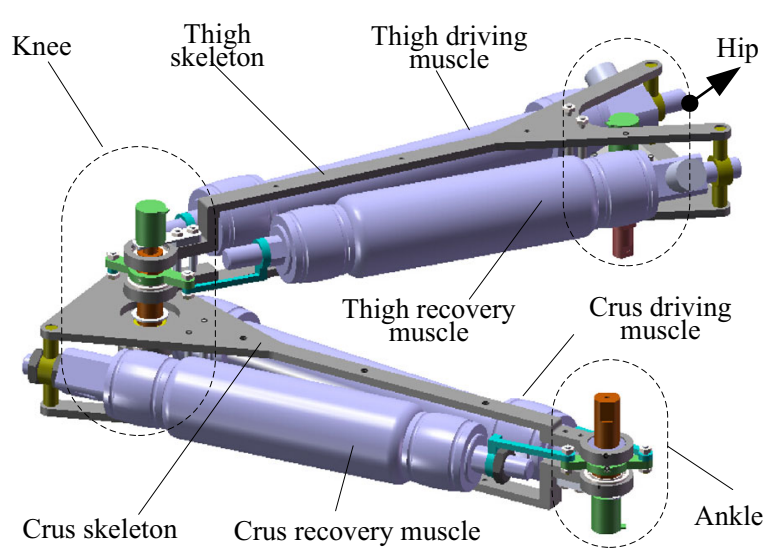

Figure 3 Structure of the hind leg
Owing to the water seal and drag reduction in water, the leg is sealed with the shells, which are made by $3 \mathrm{D}$ printing with tough resin as material. Figure 4 shows the joint dynamic seal structure, where a V-shaped seal ring is firmly set in the joint axis. The upper part of the seal ring contacts and presses the bottom face of the thigh shell during joint rotation to prevent water from entering the interior of the shell. Meanwhile, a cavity is formed along the shaft between the bearing and the shell, which forms an oil chamber, which is filled with waterproof grease that could further prevent water from entering the shell.

In the propulsive phase, frog palms are completely open to increase the area for water repulsion. This action generates the reactive force for propulsion. In the recovery phase, the palms shrink to a minimum area by concentrating the palm spokes to reduce fluid drag. To realize those functions, the design shown in Figure 5 is used. The design consists of palm spokes, steering motor, membrane, and connector for the joint shaft. One distal spoke is fixed, while the other distal spoke is rotated by the steering motor.

\subsubsection{Design of Pneumatic Circuit}

Given that the pressure proportional valve has the disadvantages of large volume and mass, high cost, slow pressurizing, and depressurizing speed, we use pneumatic highspeed switch valves to control the pressure process of the pneumatic muscles. The pneumatic circuit of the hind leg of the robot is shown in Figure 6.

The three revolute joints of each hind leg use six pneumatic muscles in total, and each pneumatic muscle is controlled by two high-speed switch valves. Thus, the entire hind limb requires 12 pneumatic high-speed switch valves. We consider the hip joint as an example to explain the working principle of the pneumatic circuit. The body and the thigh crank are connected with a joint shaft. Each

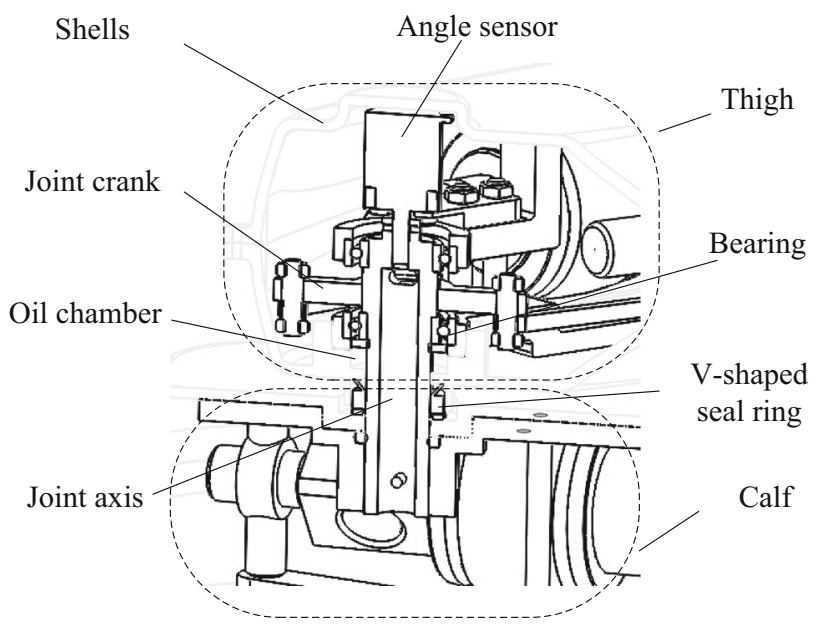

Figure 4 Structure of the joints 


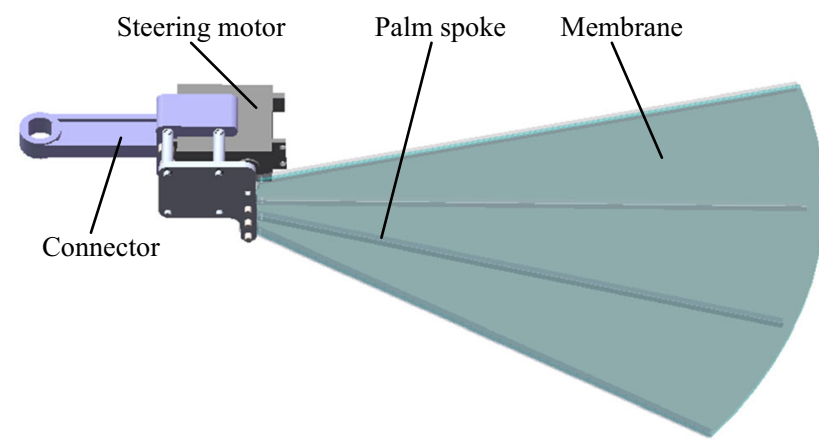

Figure 5 Palm structure

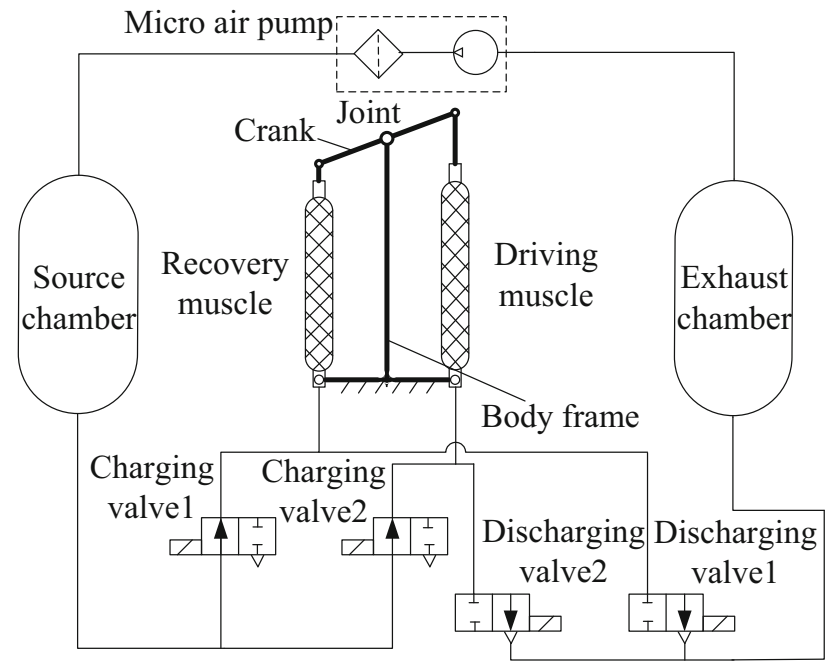

Figure 6 Pneumatic circuit of the joint

end of the crank is hinged to the muscles, and the other ends are connected to the body frame. During muscle contraction and stretching, the crank (fixed with the thigh frame) rotates relative to the body. To reach the initial position, the charging valve 1 and discharging valve 2 are open, and the discharging valve 1 and charging valve 2 are closed. The recovery muscle then starts to contract, and the driving muscle stretches until the joint rotates clockwise to the preset position (Figure 6). During the propulsive phase, the hip joint must rotate counterclockwise quickly, and thus the charging valve 1 and discharging valve 2 are close, and charging valve 1 and the discharging valve 2 are open. The recovery muscle of the hip joint then starts to stretch and the driving muscle contracts. The hip joint rotates counterclockwise quickly to a specific position.

\section{Model of the Pneumatic System}

The dynamic characteristics of the pneumatic system must be analyzed to determine the function of the musculoskeletal system of the frog-inspired robot and lay the foundation for its control system design. The pneumatic muscles used in the robot have apparent nonlinearity and hysteresis characteristics. The previous modeling of the pressure dynamic process is based on the ideal conditions, which can lead to different response results. The dynamic models of the source chamber, muscle volume, exhaust chamber, and switch valves are established to simulate the pressure process of the pneumatic system.

\subsection{Dynamic Model of the Source Chamber and Exhaust Chamber}

The source chamber is regarded as a variable mass system. The equation of the thermodynamic process can be written according to the first law of thermodynamics [25]:

$\mathrm{d} Q_{\mathrm{s}}+i_{\mathrm{s}} \mathrm{d} M_{\mathrm{s}}=\mathrm{d} U_{\mathrm{s}}+\mathrm{d} W_{\mathrm{s}}+i \mathrm{~d} M$,

where $\mathrm{d} Q_{\mathrm{s}}-$ Heat gained in the source chamber caused by the heat exchange between the inner gas and the outside world through wall, $i_{\mathrm{s}}$-Specific enthalpy of the gas flow into the source chamber, $i$-Specific enthalpy of the gas flow to the pneumatic muscle from the source chamber, $\mathrm{d} M_{\mathrm{s}}-$ Gas mass flow into the source chamber, $\mathrm{d} U_{\mathrm{s}}-\mathrm{In}$ ternal energy change of the gas in the source chamber, $\mathrm{d} W_{\mathrm{s}}$ -Expansion work done by the gas in the source chamber, $\mathrm{d} M$ - Gas mass flow into the pneumatic muscle.

According to thermodynamics, $\mathrm{d} U_{\mathrm{s}}=C_{\mathrm{v}} M_{\mathrm{s}} \mathrm{d} T_{\mathrm{s}}$, $\mathrm{d} W_{\mathrm{s}}=P_{\mathrm{s}} \mathrm{d} V_{\mathrm{s}}$ and $i \mathrm{~d} M=\left(C_{\mathrm{v}}+R\right) T_{\mathrm{s}} \mathrm{d} M$, where $C_{\mathrm{v}}$ is the constant volume specific heat of air, $R$ denotes the gas constant of air, and $\mathrm{d} T_{\mathrm{s}}$ is the temperature differential in the source chamber.

Given that the gas flow in the air pipe is faster than the heat exchange rate between gas and external environment, energy loss in the air pipe is much lesser than total gas energy. Hence, heat exchange can be ignored. Therefore, rapid pressurizing and depressurizing processes of pneumatic muscles can be regarded as adiabatic processes, such that $\mathrm{d} Q_{\mathrm{s}}=0$. The volume of the source chamber is constant, such that $\mathrm{d} V_{\mathrm{s}}=0$. If the air supply to the source chamber is disregard, then $\mathrm{d} M_{\mathrm{s}}=0$. Therefore, the gas thermal process in the source chamber can be simplified as follows:

$\mathrm{d} p_{\mathrm{s}}=-\frac{k R T_{\mathrm{s}} \mathrm{d} M}{V_{\mathrm{s}}}$,

where $T_{\mathrm{s}}$-Temperature in the source chamber, $p_{\mathrm{s}}$-Pressure in the source chamber, $R-$ Gas constant of air, $287 \mathrm{~J} \cdot \mathrm{kg} / \mathrm{K}, K$-Specific heat ratio of air, $K=1.4$.

The relationship between temperature and pressure in the adiabatic process is represented as

$\frac{T_{\mathrm{S}}}{T_{0}}=\left(\frac{p_{\mathrm{S}}}{p_{0}}\right)^{\frac{k-1}{k}}$, 
where $T_{0}$-Initial temperature in the source chamber, $p_{0}-$ Initial pressure in the source chamber.

Similarly, the thermal process of the pressurizing process in the exhaust chamber is derived as

$k R T \mathrm{~d} m=V_{\mathrm{e}} \mathrm{d} p_{\mathrm{e}}$,

where $T$-Initial temperature in the pneumatic muscle, $p_{\mathrm{e}}$-Pressure in the exhaust chamber, $V_{\mathrm{e}}-$ Volume of the exhaust chamber, $\mathrm{d} m$-Exhausted air from the pneumatic muscle.

\subsection{Dynamic Model of the Pneumatic Muscle}

When the pneumatic muscle is assumed as a cylinder and the noncylindrical joint at both ends of the muscle is disregarded, the pneumatic muscle structure can be modeled as shown in Figure 7. In the figure, $\theta$ is the angle between the braided thread and central axis, and $D$ is the pneumatic muscle diameter. Muscle volume can be expressed as follows [26]:

$V=\frac{b_{0}^{2}-L^{2}}{4 \pi n^{2}} L$,

where $L$-Pneumatic muscle length, $n-$ Number of loops of braided thread in the muscle, $b_{0}$-Length of the nonretractable braided thread, $V$-Internal volume of the muscle.

According to Eq. (1), the pressurizing process of the muscle can be simplified as

$k R T_{\mathrm{S}} \mathrm{d} M=k P \mathrm{~d} V+V \mathrm{~d} P$.

The thermal process of muscle depressurizing is

$-k R T \mathrm{~d} m=k P \mathrm{~d} V+V \mathrm{~d} P$,

where $P$-Pressure in pneumatic muscle, $V$-Volume of pneumatic muscle.

\subsection{Dynamic Model of the Pneumatic System}

The pneumatic system in this paper uses the switch valve, which has a simple structure and digital control signal. The flow characteristics of the pneumatic system mainly
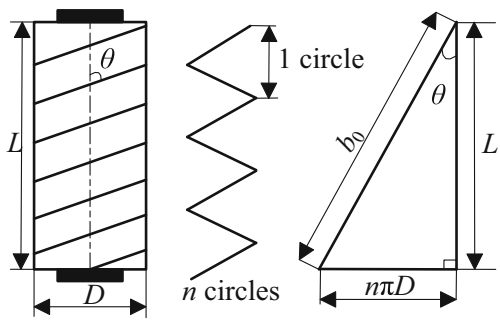

Figure 7 Structure model of pneumatic muscles depend on the valve $[27,28]$. Thus, we use the high-speed switch valve MHJ9-LF manufactured by FESTO. The valve Sonic conductance $(C)$ is $0.4 \mathrm{~L} / \mathrm{s} \cdot \mathrm{bar}$, and the critical pressure ratio $(b)$ is 0.38 . The volume flow through the valve can be solved by the following equation:

$Q_{\mathrm{m}}=C\left(P_{1}+P_{0}\right) \sqrt{\frac{T_{0}}{T_{1}}} \omega(\sigma, b)$,

$\omega(\sigma, b)=\left\{\begin{array}{cc}1 & \sigma \leq \mathrm{b}, \\ \sqrt{1-\left(\frac{\sigma-b}{1-b}\right)^{2}} & \sigma \leq \mathrm{b},\end{array}\right.$

$\sigma=\frac{P_{2}+P_{0}}{P_{1}+P_{0}}$

where $P_{0}$-Atmospheric pressure in standard condition, $P_{1}$-Upstream pressure, $P_{2}$-Downstream pressure, $T_{0}$ Gas temperature in the standard state.

As the gas cylinder connects six branch pipes for the muscles from one main pipe simultaneously, the gas flow at saturation, and the gas flow in the main pipe can be obtained through the continuity equation:

$Q=\pi r^{2} v$

where $Q$-Main pipe flow rate, $r$-Pipe radius, $v-$ Flow velocity in the pipe.

On the basis of Eqs. (2), (3), (5), (6), and (8), a simulation model of the pneumatic system of the robot is established to simulate the dynamic characteristics of the process of pneumatic muscle pressurizing, as shown in Figure 8.

The saturation term is added in the simulation process because of the calculated maximum flow rate in the main pipe. The muscle length is derived from the joint angle. With muscle length and initial pressure from the source chamber, the pressurizing processes in the chambers and muscles can be simulated, and the rationality of the established model can be verified by comparing the results to lay the foundation for future designs of robot control systems.

\section{Simulations and Experiments}

The prototype of the frog-inspired robot is shown in Figure 9. This prototype is based on previously introduced designs. In the experiments, the source chamber was connected to a $6 \mathrm{~mm}$ pipe (with inner diameter of $4 \mathrm{~mm}$ ) to serve as the high pressure source. Then, the pipe was divided into six branches to connect to the switch valves and muscles with $4 \mathrm{~mm}$ pipes (with inner diameter of $2.5 \mathrm{~mm}$ ). The parameters in the robot are shown in Table 1. 
Figure 8 Simulation model of the pneumatic system

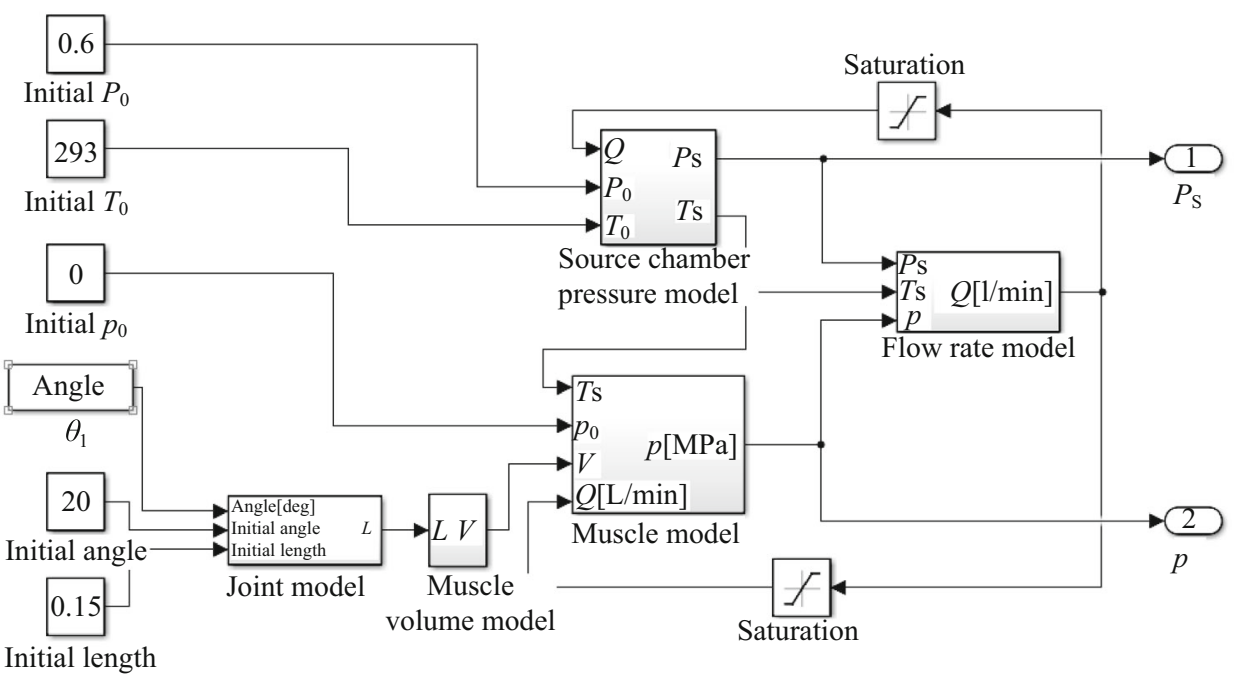

Initial length

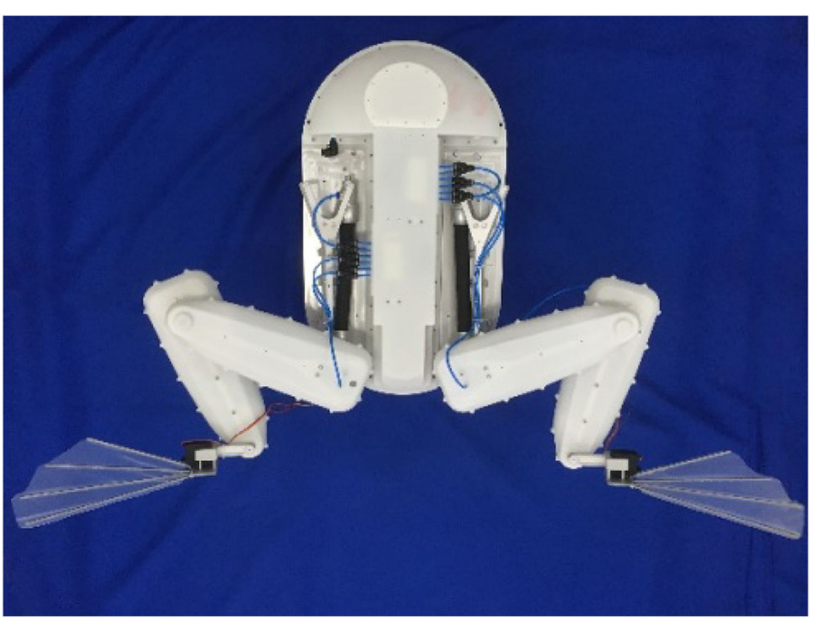

Figure 9 Prototype of frog-inspired robot

Table 1 Parameters of the frog-inspired robot

\begin{tabular}{ll}
\hline Parameter & Value \\
\hline Body length $l_{0} / \mathrm{m}$ & 0.590 \\
Body width $w_{0} / \mathrm{m}$ & 0.340 \\
Thigh length $l_{1} / \mathrm{m}$ & 0.280 \\
Crus length $l_{2} / \mathrm{m}$ & 0.280 \\
Palm length $l_{3} / \mathrm{m}$ & 0.336 \\
Palm area $S / \mathrm{m}^{2}$ & 0.0335 \\
Total mass $m / \mathrm{kg}$ & 12 \\
Displacement $V_{\mathrm{d}} / \mathrm{L}$ & 19 \\
\hline
\end{tabular}

Figure 10 shows the experiment platform. A simple circuit is established to transfer the high pressure from the source chamber to the muscles via the high-speed switch valves. The switch valves are controlled by the control card (Arduino Mega 2560).

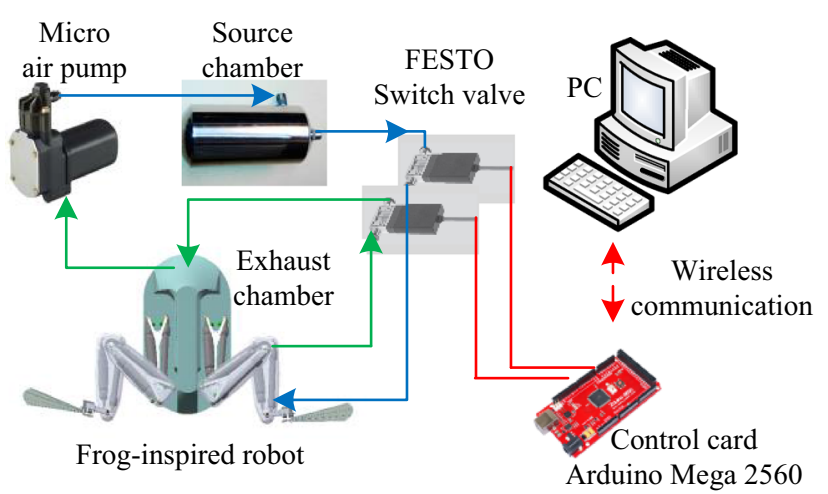

Figure 10 Experiment platform

The swimming cycle is divided into the propulsive and recovery phases according to body velocity. The leg motions are powerful and rapid in the propulsive phase, while the legs recover slowly during the recovery phase. Therefore, the experiments and simulations on the hind leg extensions were conducted to test the muscle capacity to drive the system. The pressures in the pneumatic muscles were controlled by the high-speed switch valves with open loop control.

\subsection{Swimming Experiments}

For the validation of the designed frog-inspired robot system and experimental data collection, an experiment wherein the leg extension had no load and robot swimming experiment were conducted. In the experiments, the recovery muscle was removed and replaced with a light spring for recovery to eliminate the interaction of the recovery muscle which response differently according to control strategy. The source chamber was pressurized to six bars in advance, and the muscle was aerated through the 


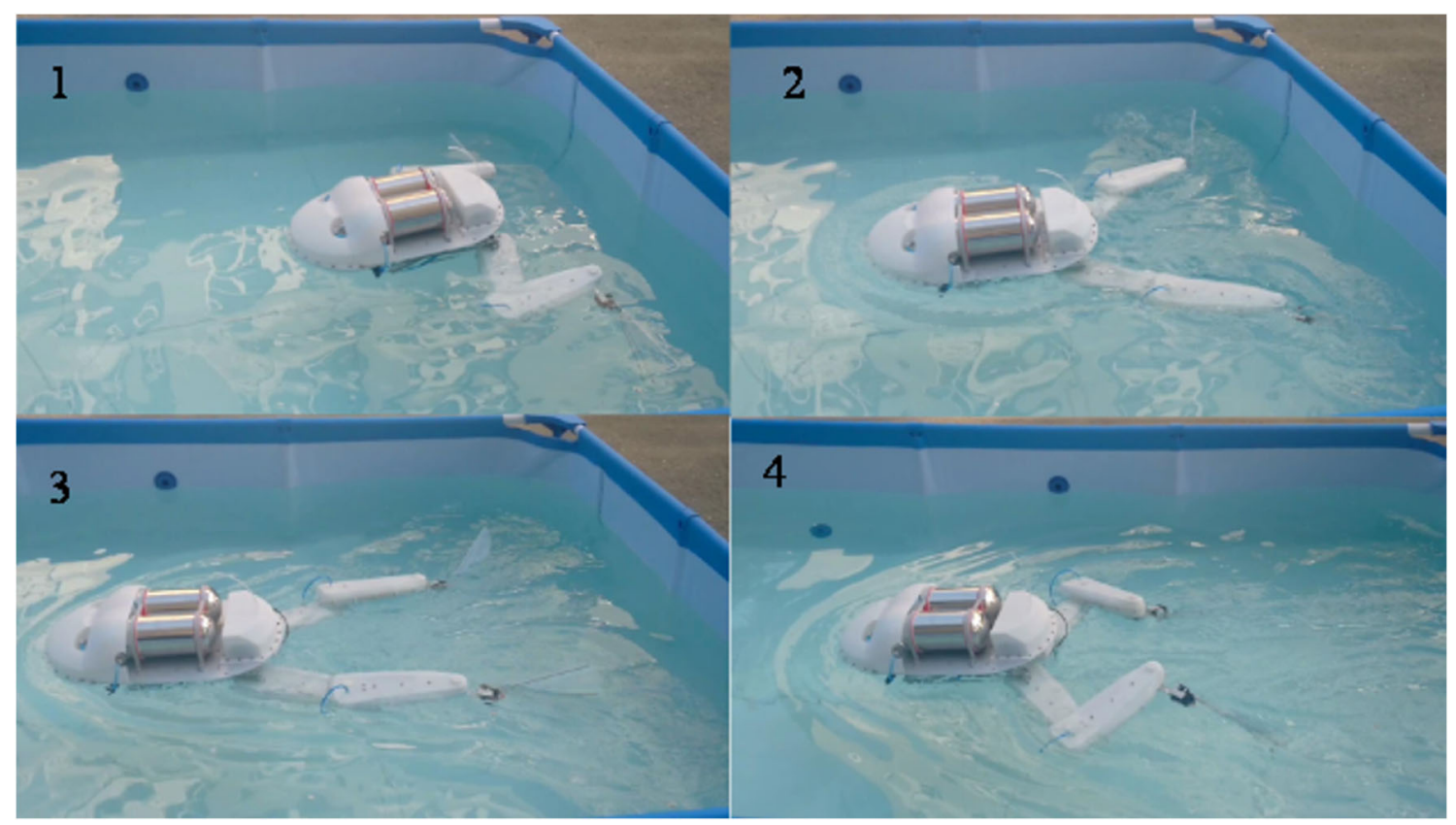

Figure 11 Swimming experiment under water

switch valves. The open loop control was applied to the muscles, which were pressurized for one second and then released for one second. The swimming experiment for one period was conducted to verify the robot design, as shown in Figure 11. The results of the joint angle are shown in Figure 12.

Each joint had a delay of about $0.1 \mathrm{~s}$, because of the influence of the delay in the pneumatic system and mechanical clearance in the joints. The joint data would be used in the subsequent simulations to compute the pressure dynamic process according to previously established pneumatic model.

\subsection{Simulation of the Pneumatic System}

The experiments in the air were conducted, and the frog body was fixed on a base. The joint angles were measured by the sensors in the robot, and the pressures were measured by the sensors in the external circuits. With the pneumatic muscle volume data derived from the joint angle results, the dynamic model built in MATLAB/Simulink was able to simulate the pressurizing processes in the muscle volume and source chamber. The pressurizing process from the experiments and that of the simulations were compared (Figure 13) to validate the model of the pneumatic system. The pressurizing processes in the hip muscle from simulation matched well with the experiment. Figure 14 shows the results in the source chamber. Compared with the small muscle volume, which rendered the noise evident, the pressure drop is smaller because of its large chamber capacity. Finally, simulation results are

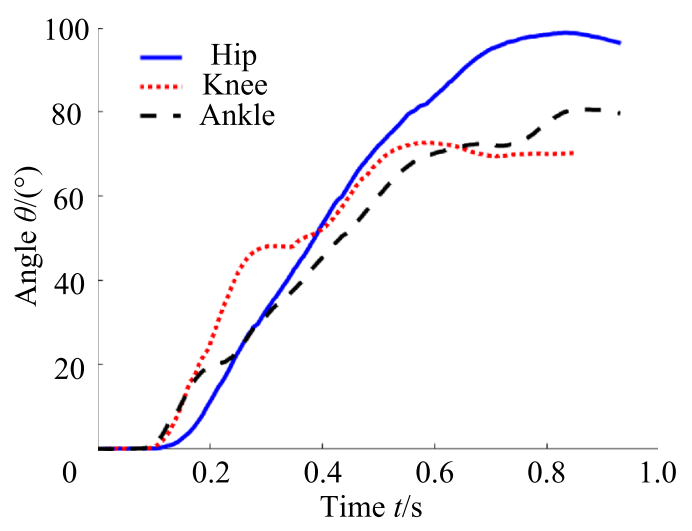

Figure 12 Joint angles from the experiment

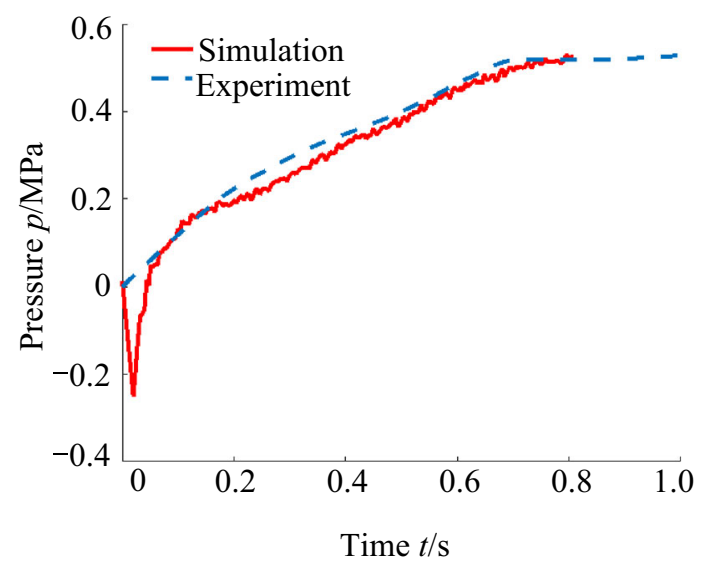

Figure 13 Results of the hip muscle pressure 


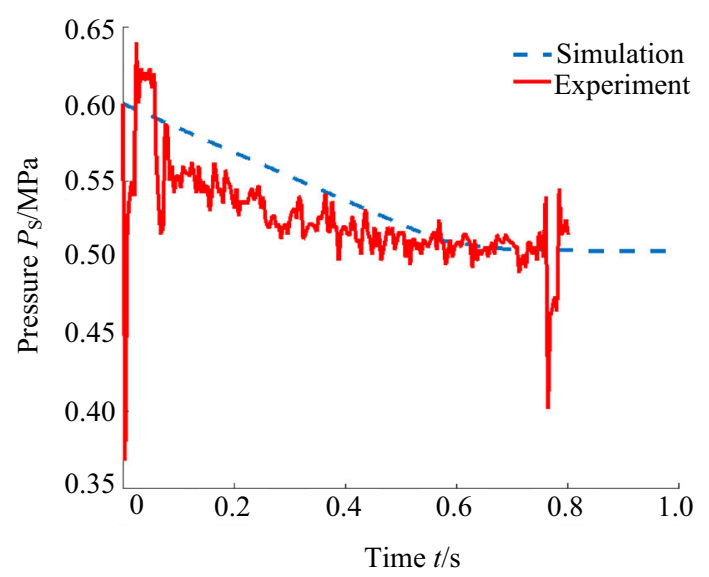

Figure 14 Results of the source chamber pressure

consistent with the experiment and converged to the same end. These results verified the feasibility of the pneumatic model built in this paper.

According to Eq. (8), the aeration volumes of each joint can be obtained by integrating the flow rate during muscle pressurizing. The aeration volumes at standard state were computed in the simulation. The aeration volume at the hip muscle is $0.455 \mathrm{~L}$ and that at the knee and ankle muscle is $0.401 \mathrm{~L}$. The aeration volumes are the key factors that verify the design of the pneumatic system.

\subsection{Analysis on the Pressurizing Process}

The response speed of the pneumatic muscle reflects the performance of the driving capacity and is a key factor to simulate the musculoskeletal system $[29,30]$. The response speed is mainly restricted by the pressurizing process. The simulations with different source pressures, muscle volumes, and flow saturations in the pneumatic circuit were calculated to analyze the factors of muscle performance. Therefore, the pressure response in the pneumatic muscle can be obtained.

The simulation results of the pressurizing process in the hip-driving muscle was influenced by different initial source pressures (Figure 15). The branches from the main pipe were considered during the calculation of the maximum flow rate in the main pipe through Eq. (9). The saturation for each branch pipe connected with switch valves and muscles were set at $45 \mathrm{~L} / \mathrm{min}$ after the average distribution of the flow rate. The final steady muscle pressures varied with source pressures (Figure 15(a)). However, the pressurizing process was identical before the balanced state. The pipe saturation limited the flow rate through the valves and muscles, as normal sources of pressures in the simulations generated a flow rate higher than $45 \mathrm{~L} / \mathrm{min}$. Therefore, at pipe saturation, the initial source pressure has minimal influence on the muscle pressurizing process.

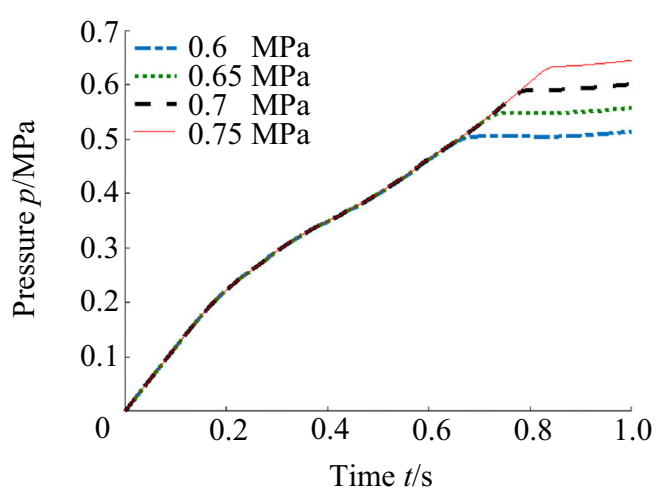

(a) Hip muscle pressure with saturation

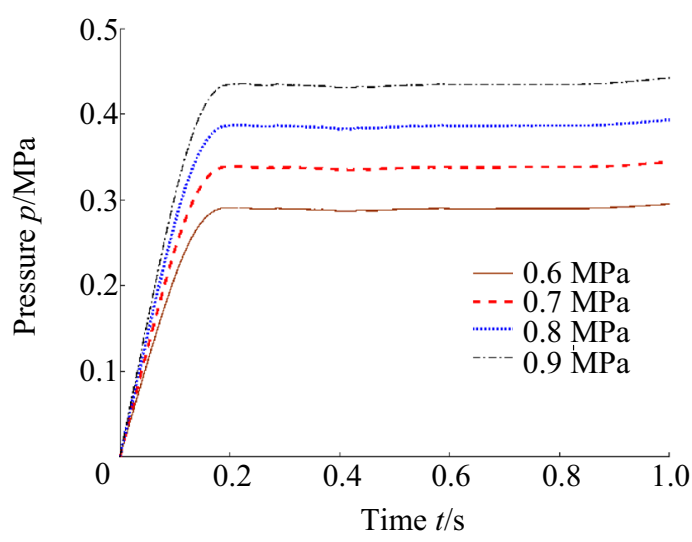

(b) Hip muscle pressure without saturation

Figure 15 Pressure responses under various sources

Figure 15(b) shows the results without the pipe saturation. After removing the saturation, the pressure response shortened and fell within $0.2 \mathrm{~s}$, thus illustrating that the source pressure promotes the flow in the pipe.

Figure 16 shows the simulation results of the pressurizing process in the hip joint muscle at an initial source pressure of 6 bar and $100 \%$ muscle volume constant. To estimate the influence of each muscle volume, the $100 \%$ muscle volume constant was set as the maximum of the muscle volume during contraction, and $0 \%$ muscle volume constant was set as the original muscle volume before pressurizing. Figure 16(a) shows the results with saturation and without saturation. Although a small volume required low-pressure air to receive faster response, the difference between the maximum and minimum volumes during contraction was relatively minimal at small volumes compared with those at the large initial muscle volume. Therefore, the results reflected the minimal influence of the variation of muscle volumes.

Figure 17 shows the simulation results of the pressurizing process in the hip joint muscle at initial source pressure of 6 bar and $100 \%$ muscle volume constant. At 


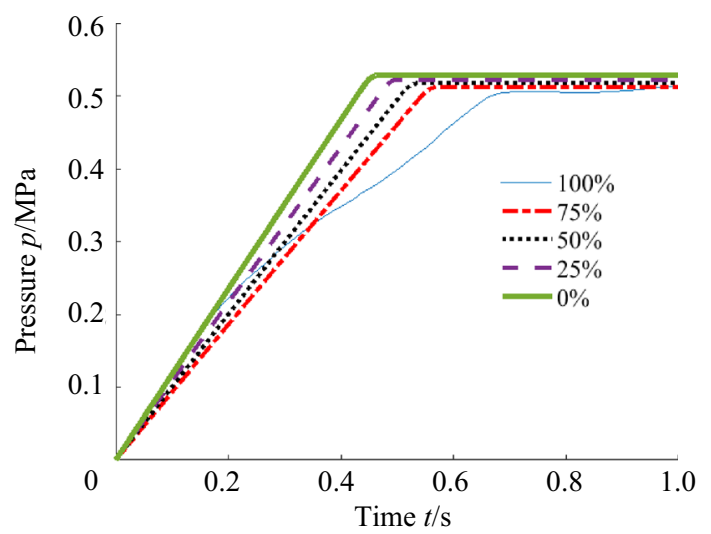

(a) Hip muscle pressure with saturation

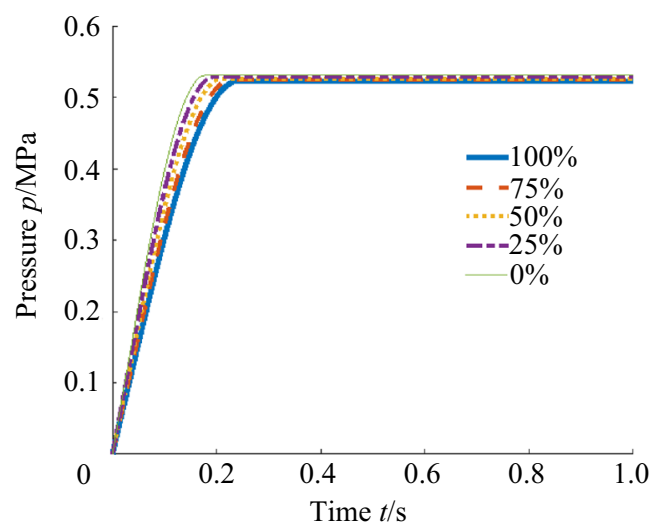

(b) Hip muscle pressure without saturation

Figure 16 Pressure response under various muscle volumes

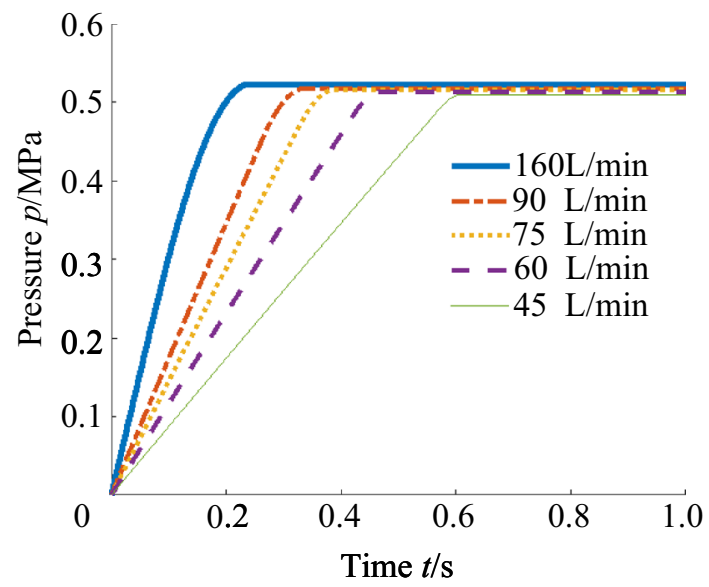

Figure 17 Pressure response under various saturations

different saturations in the pipe, the pressurizing processes showed different response speeds.

The flow rate limitation in the pneumatic circuits is the main factor that controls the pressure response in the muscle. Therefore, in the robot design, extending the main pipe diameter and number of main pipes is an effective way to improve the pressurizing speed. The results of the experiments and simulations verified the pneumatic model of the frog-inspired robot.

\section{Conclusions}

(1) A frog-inspired robot is designed to mimic the frog swimming based on its three DOFs in the leg and the pneumatic muscles serving as the joint driver. The prototype can perform untethered swimming which are supported by the integrated pneumatic, control, and communication systems in the body.

(2) The pneumatic system is modeled based on flow rate characteristics during the pressurizing and depressurizing of the muscles to analyze the nonlinearity of the driver. The pneumatic model is proved reasonable by the simulations, and the findings is the base for the controller design in the near future.

(3) The experiments and simulation of the robot indicate that the robots driven by pneumatic muscles are feasible and the flow rate is the main factor for the quick response of the pneumatic system.

Open Access This article is distributed under the terms of the Creative Commons Attribution 4.0 International License (http://crea tivecommons.org/licenses/by/4.0/), which permits unrestricted use, distribution, and reproduction in any medium, provided you give appropriate credit to the original author(s) and the source, provide a link to the Creative Commons license, and indicate if changes were made.

\section{References}

1. Z J Yu, Z Q Zheng, X G Yang, et al. Dynamic analysis of propulsion mechanism directly driven by wave energy for marine mobile buoy. Chinese Journal of Mechanical Engineering, 2016, 29(4): 710-715.

2. B Tondu, P Lopez. The McKibben muscle and its use in actuating robot-arms showing similarities with human arm behavior. Industrial Robot, 1997, 24(6): 432-439.

3. J Guo. Optimal measurement strategies for target tracking by a biomimetic underwater vehicle. Ocean Engineering, 2008, 35(5): 473-483.

4. Y L Hou, X Z Hu, D X Zeng, et al. Biomimetic shoulder complex based on 3-pss/s spherical parallel mechanism. Chinese Journal of Mechanical Engineering, 2015, 28(1): 29-37.

5. Y W Wang, Z L Wang, Jian Li. Research development and tendency of biomimetic robot fish. Machine Design and Research, 2011, 27(2): 22-25, 32.

6. M S Triantafyllou, G S Triantafyllou. An efficient swimming machine. Scientific American, 1995, 272(3): 64-71.

7. C zhou, M Tan, Z Q Cao. Kinematic modeling of a bio-inspired robotic fish. IEEE International Conference on Robotics and Automation, Pasadena, CA, USA, May 19-23, 2008: 695-699. 
8. L Shi, S X Guo, S L Mao, et al. Development of a lobsterinspired underwater micro robot. International Journal of Advanced Robotic Systems, 2013, 10(1): 257-271.

9. J Ayers. Underwater walking. Arthropod structure \& development, 2004, 33(3): 347-360.

10. A P Thomas, M Milano, K Fischer, et al. Synthetic jet propulsion for small underwater vehicles. Proceedings of the IEEE International Conference on Robotics and Automation. Barcelona, Spain, April 18-22, 2005: 181-187.

11. P V Alvarado, S Chin, W Larson. A soft body under-actuated approach to multi degree of freedom biomimetic robots: A stingray example. Proceedings of the 2010 3rd IEEE RAS \& EMBS International Conference on Biomedical Robotics and Biomechatronics, Tokyo, Japan, September 26-29, 2010: 473-478.

12. M Wang, X Z Zang, J Z Fan, et al. Biological jumping mechanism analysis and modeling for frog robot. Journal of Bionic Engineering, 2008, 5: 181-188.

13. J M Gal, R W Blake. Biomechanics of frog swimming: I. estimation of the propulsive force generated by Hymenochirus boettgeri. Journal of Experimental Biology, 1988, 138(1): 399-411.

14. C T Richards. Kinematics and hydrodynamics analysis of swimming anurans reveals striking inter-specific differences in the mechanism for producing thrust. Journal of Experimental Biology, 2010, 213(4): 621-634.

15. S Nauwelaerts, P Aerts, K D'août. Speed modulation in swimming frogs. Journal of Motor Behavior, 2001, 33(3): 265-272.

16. J Pandey, N S Reddy, R Ray, et al. Biological swimming mechanism analysis and design of robotic frog. 2013 IEEE International Conference on Mechatronics and Automation, Takamatsu, Japan, August 4-7, 2013: 1726-1731.

17. D A Núñez-Altamirano, I Juárez-Campos, L Márquez-Pérez, et al. Description of a propulsion unit used in guiding a walking machine by recognizing a three-point bordered path. Chinese Journal of Mechanical Engineering, 2016, 20(6): 1157-1166.

18. J T Lei, H Y Yu, T M Wang. Dynamic bending of bionic flexible body driven by pneumatic artificial muscles(PAMs) for spinning gait of quadruped robot. Chinese Journal of Mechanical Engineering, 2016, 29(1): 11-20.

19. V T Jouppila, S A Gadsden, G M Bone. Sliding mode control of a pneumatic muscle actuator system with a PWM strategy. International Journal of Fluid Power, 2014, 15(1): 19-31.

20. H P Ren, J T Fan. Adaptive backstepping slide mode control of pneumatic position servo system. Chinese Journal of Mechanical Engineering, 2016, 29(5): 1003-1009.

21. G L Shi, W Shen. Hybrid control of a parallel platform based on pneumatic artificial muscles combining sliding mode controller and adaptive fuzzy CMAC. Control Engineering Practice, 2013, 21: 76-86.

22. M Sorli, L Gastaldi, E Codina, et al. Dynamic analysis of pneumatic actuators. Simul. Pract. Theory, 1999: 589-602.

23. A Ilchmann, O Sawodny, S Trenn. Pneumatic cylinders: modelling and feed-back force-control, International Journal of Control, 2006, 79(6): 365-78.
24. H Ali, S Noor, S M Bashi, et al. A review of pneumatic actuators (modeling and control). Australian Journal of Basic and Applied Sciences, 2009, 3(2): 440-454.

25. Y Chen, J F Zhang, C J Yang, et al. Design and hybrid control of the pneumatic force-feedback systems for Arm-Exoskeleton by using on/off valve. Mechatronics, 2007, (17): 325-335.

26. C P Chou, B Hannaford. Measurement and modeling of Mckibben pneumatic artificial muscles. IEEE Transactions on Robotics and Automation, 1996, 12(1): 90-102.

27. B S Kang, C S Kothera, B K S Woods, et al. Dynamic modeling of mckibben pneumatic artificial muscles for antagonistic actuation. IEEE International Conference on Robotics and Automation, Kobe, Japan, May 12-17, 2009: 182-187.

28. Y P Ju, H Liu, Z Y Yao, et al. Fluid-structure interaction analysis and lifetime estimation of a natural gas pipeline centrifugal compressor under near-choke and near-surge conditions. Chinese Journal of Mechanical Engineering, 2015, 28(6): 1261-1268.

29. Ž Šitum, P Trslić, D Trivić, et al. Pneumatic muscle actuators within robotic and mechatronic systems. Proceedings of International Conference Fluid Power, Maribor, Slovenija, September 17-18, 2015: 175-188.

30. I Veneva, B Vanderborght, D Lefeber, et al. Propulsion system with pneumatic artificial muscles for powering ankle-foot orthosis. Journal of Theoretical and Applied Mechanics, 2013, 43(4): 3-16.

Ji-Zhuang Fan, born in 1976, is currently an associate professor and a PhD candidate supervisor at State Key Laboratory of Robotics and System, Harbin Institute of Technology, China. He received his $\mathrm{PhD}$ degree from Harbin Institute of Technology, China, in 2008. His main research interests include mechachonics engineering and bionic robotics. E-mail: fanjizhuang@hit.edu.cn

Wei Zhang, born in 1988, is currently a $\mathrm{PhD}$ candidate at State Key Laboratory of Robotics and System, Harbin Institute of Technology, China. He received his master degree from Harbin Institute of Technology, China in 2012. His research interests include mechanical design and bionic robotics. E-mail: zhangwei0229@aliyun.com

Peng-Cheng Kong, born in 1992, received his master degree from Harbin Institute of Technology, China, in 2016. E-mail: kongpch@qq.com

He-Gao Cai, is currently a professor and a $\mathrm{PhD}$ candidate supervisor at State Key Laboratory of Robotics and System, Harbin Institute of Technology, China. E-mail: hgcai@hope.hit.edu.cn

Gang-Feng Liu, born in 1980, is currently a lecturer at State Key Laboratory of Robotics and System, Harbin Institute of Technology, China. He received his $\mathrm{PhD}$ degree from Harbin Institute of Technology, China, in 2010. His main research interests include robotics, teleoperation and space manipulator technology. 